\title{
Prevalencia y factores asociados a sobrepeso y obesidad infantil en escuelas públicas y privadas de Tegucigalpa, Honduras
}

\author{
Prevalence of pediatric overweight and obesity and associated \\ factors in public and private schools in Tegucigalpa, Honduras
}

\begin{abstract}
Objective: To compare the factors associated with overweight and obesity in children from public and private schools in the Kennedy colony, Tegucigalpa, Honduras. Materials and methods. We studied 357 schoolchildren between 6 and 11 years old. A survey was used to assess nutrition, food frequency, eating habits and practices, and physical activity. Students with overweight and obesity secondary to a basic pathology were excluded. The information was analyzed using Epi-info. We conducted a univariate and multivariate analyzes. Results: We found that $18 \%$ of the students were obese and 18\% were overweight. Students from private schools were more frequently overweight and obese $(46.3 \%)$ than those in public schools $(33.2 \%)$. Fifty-three percent of public school students and $36.3 \%$ of private schools consumed homemade snacks. Over half $(58.5 \%)$ of public school students and $68.7 \%$ of private schools reported doing some kind of outdoor physical activity. Conclusion: Childhood overweight and obesity are prevalent health problems. Private school students, in our sample, were more overweight and obese than public school students. Physical activity performed by school children in public and private schools was adequate in most cases.

Key Word: Child Nutrition; Eating; Diet, Pediatric Obesity; Overweight
\end{abstract}

\section{INTRODUCCIÓN}

Según la Organización Mundial de la Salud (OMS) el sobrepeso y la obesidad se definen como una acumulación anormal o excesiva de grasa que supone un riesgo para la salud'. Presentando un incremento dramático a nivel mundial en las últimas tres décadas, 1.000 millones de personas en el mundo tienen sobrepeso y obesidad. Esta prevalencia tiene grandes variaciones geográficas con valores que van desde el $5 \%$ para Asia y África, hasta el $20 \%$ en América ${ }^{2-5}$. A nivel mundial cerca del $10 \%$ de los niños en edad escolar presentan sobrepeso $0^{6,7}$ por lo que la OMS, considera a la obesidad como la "Epidemia del siglo $\mathrm{XXI}^{\prime \prime 4}$. En el año 2012 la prevalencia de niños menores de cinco años con sobrepeso aumentó a 30 millones en países

\begin{abstract}
Alejandro Ramírez-Izcoa', Luis Enrique Sánchez-Sierra ${ }^{\top}$, Carlos Mejía-Irías' ${ }^{1}$, Allan Iván Izaguirre González ${ }^{1,2,}$ Cindy Alvarado-Avilez' ${ }^{1}$, Rosaura Flores-Moreno ${ }^{1}$, Kyrian Yareny Miranda', Claudia M-Díaz', Videlda Grissel Aguilar ${ }^{1}$, Elena E. Rivera ${ }^{3}$.
\end{abstract}

\footnotetext{
1. Facultad de Ciencias Médicas, Universidad Nacional Autónoma de Honduras (UNAH), Tegucigalpa, Honduras 2. Centro Integral en Salud (CIS) - Amigos de las Américas (ONG), Región \# 7, Trojes, El Paraíso, Honduras.

3. Departamento de Nutrición, Facultad de Ciencias Médicas, UNAH
}

Dirigir correspondencia a: Alejandro Ramírez-lzcoa. Facultad de Ciencias Médicas, Universidad Nacional Autónoma de Honduras (UNAH), Tegucigalpa, Honduras (+504) 3178-6135, Tegucigalpa, Honduras. E-mail: alejandro.izcoa@gmail.com.

Este trabajo fue recibido el 17 de diciembre de 2016. Aceptado con modificaciones el 20 de abril de 2017 y aceptado para ser publicado el 09 de mayo de 2017.

en desarrollo, mientras que en los países desarrollados esa cifra fue de 10 millones $^{6,7}$. En América Latina, se calcula que $7 \%$ de los menores de 5 años de edad (3,8 millones) tienen sobrepeso u obesidad ${ }^{3}$. En Honduras, en el año 2000 se estudió la población escolar de Tegucigalpa y reportó, acorde al IMC que el $2 \%$ de estos niños tenían bajo peso, un $14 \%$ sobrepeso y un $6 \%$ obesidad $^{5}$.

El término malnutrición incluye tanto las carencias como los excesos o desequilibrios en la ingesta de energía, proteínas y otros nutrientes. Por lo que el sobrepeso y la obesidad se definen como el exceso de peso corporal a expensas fundamentalmente de la masa grasa, siendo consideradas como enfermedades crónicas no transmisibles de inicio temprano como la infancia y adolescencia, de 
origen multifactorial donde intervienen factores genéticos, ambientales y conductuales, que incrementan el riesgo de morbilidad por enfermedades crónicas no transmisibles acompañantes (ECNT) $)^{8-12}$. En la etapa escolar el mundo del niño se amplía, al igual que las oportunidades de comer fuera del ambiente familiar, y se expone a diferentes alimentos y diversas formas de prepararlos, con distintos horarios y lugares, lo que facilita el consumo de una importante cantidad de alimentos sin supervisión familiar, principalmente fuera del hogar, lo cual puede facilitar el consumo de alimentos densamente energéticos y desprovistos de micronutrientes ${ }^{13,14}$.

El plan de acción contra el sobrepeso y la obesidad se debe centrar en la niñez por las siguientes razones: son enfermedades de inicio temprano, poseen mayor riesgo de ser acompañadas de otras enfermedades crónicas no transmisibles, y porque los hábitos alimentarios son adquiridos durante la niñez $z^{2}$. Por lo anteriormente expuesto, nos propusimos como objetivo determinar la prevalencia de sobrepeso y obesidad en niños de escuelas públicas y privadas de la colonia Kennedy en Tegucigalpa (durante mayo de 2016) y comparar estos resultados con los factores asociados.

\section{SUJETOS Y MÉTODOS}

Diseño: Estudio descriptivo transversal, estratificado aleatorio.

De un universo de 2.144 escolares entre 6 y 11 años de edad se estudió a 357 niños. Para calcular la muestra se utilizó la fórmula definida por Sampieri ${ }^{15}$ :

$$
n=\frac{\mathrm{N} \times \mathrm{Z}^{2} \times \mathrm{p} \times \mathrm{q}}{\mathrm{d}^{2} \times(\mathrm{N}-1)+\mathrm{Z}^{2} \times \mathrm{p} \times \mathrm{q}} ; n=320 \times 10 \%=357
$$

dónde $\mathrm{N}$ es el total de población (2.142); $Z^{2}: 1,96$ ya que la seguridad es del $95 \%$; p: probabilidad de que un evento ocurra $=0,5 ;$ q: 1 -p probabilidad que un evento no ocurra $=0,5 ;$ d: error muestral deseado 5\%. A su vez se calculó una fracción constante para realizar la estratificación del muestreo para tener una muestra representativa de cada centro educativo, según fórmula de Kish ${ }^{15}$ :

$$
k s h=\frac{\mathrm{n}}{N h} ; k s h=\frac{357}{2144} ; f h=0.1674
$$

donde $\left(N_{h}\right)$ es el total de la población y (fh) el resultado del coeficiente de Kish. Posteriormente se calculó la muestra de cada subconjunto $(n h)$ con la multiplicación de $\left(N_{h}\right)\left(f_{h}\right)=n_{h}$. Los subconjuntos se constituyeron por 6 escuelas con número diferente de estudiantes. Tres escuelas eran públicas y tres privadas, cuyas poblaciones y muestra al calcular estratos fueron las siguientes: Escuela John F. Kennedy 704 (116); Dr. Esteban Mendoza 700 (117), Gral. Oswaldo López Arellano 300 (50); Intercontinental School 250 (42); Gran Comisión 93 (15); Evangélico Luz y Vida 99 (17).

\section{Evaluación nutricional}

Se procedió a utilizar un instrumento tipo encuesta basado en el estudio de factores de riesgo nutricional empleado en Chile por Lera y et.al ${ }^{16}$. Se utilizaron medidas antropométricas (peso en kilogramos y talla en metros), las cuales se obtuvieron utilizando una balanza digital operada con batería alcalina (Health o meter ${ }^{\circledR} 822 \mathrm{KL}$, precisión $0,05 \mathrm{~kg})$, colocada en una superficie plana y sólida. Se midió la talla colocando al escolar en posición anatómica, con talones unidos y puntas separadas por un ángulo de $45^{\circ}$, en posición erecta, con región posterior apoyada hacia el tallímetro, y cabeza en plano Frankfort, con pies descalzos y con la menor ropa posible. Para calcular el índice de masa corporal, el percentil y diagnóstico nutricional, se utilizó el software AnthroPlus de la OMS para niños de 5 a 19 años.

\section{Frecuencia de Consumo y Hábitos y prácticas alimentarias}

Se utilizó el test de Coromoto-Nava en $2011^{17}$. Se evaluó la ingesta de alimentos diarios (rara vez o nunca, 1 , 2-3, 4-5 veces al día). Ingesta de bebidas, frutas, verduras, carbohidratos, carnes, golosinas (porciones, días a la semana, lugar de procedencia).

\section{Actividad física}

Se utilizó el test clínico empleado por Godard C. y et.al. ${ }^{18}$ Se midió el descanso/sueño (acostado: $(<8,8-12,>12$ hr), sentado: $(<6,6-10,>10 \mathrm{hr})$, caminando $(<5,5-15,>15$ cuadras), juegos al aire libre ( $<30,30-60,>60 \mathrm{~min})$ y ejercicio $(<2,2-4,>4 \mathrm{hr})$. Todos los test fueron adaptados a nuestro medio y a su vez validados por un grupo de expertos (metodología de investigación, especialista en nutrición), mediante una prueba piloto, los cuales no se incluyeron en él estudio. En base a esto, se diseñó una encuesta, con preguntas abiertas y cerradas. Se excluyeron escolares que rechazaron participar en el estudio, sobrepeso y obesidad secundaria a una patología de base (metabólica o endocrinológica), y con presencia de edema.

\section{Análisis estadístico}

La información recolectada fue ingresada en una base de datos, diseñada con el programa Epilnfo ${ }^{\mathrm{TM}}$ versión 7.1.4.0 (CDC, Atlanta) y Microsoft Office Excel 2016, seguido del análisis univariado y bivariado. Los resultados se presentan como frecuencias, porcentajes, rangos y promedios.

\section{Consideraciones éticas}

El protocolo contó con la aprobación del Comité de Ética en Investigación Biomédica (CEIB), de la Facultad de Ciencias Médicas (FCM), Universidad Nacional Autónoma de Honduras (UNAH). Se solicitó Se solicitó permiso institucional a las autoridades de cada centro escolar.

Dificultades metodológicas

Algunos escolares no presentaron los consentimientos informados en el primer abordaje. 


\section{RESULTADOS}

Se evaluaron 357 niños en edad escolar de escuelas públicas y privadas de la colonia Kennedy. Las características sociodemográficas estudiadas se representan en la tabla 1.

$\mathrm{Al}$ evaluar el índice de masa corporal para la edad según los percentiles de la OMS se encontró que $18 \%$ de los escolares tanto en escuelas públicas y privadas presentaron obesidad y $18 \%$ presentaron sobrepeso, mientras $54 \%$ tenían adecuado índice de masa corporal (IMC) para la edad, por el contrario $7 \%$ tenían riesgo de bajo peso y $3 \%$ presentaron bajo peso (Gráfico 1).

Al comparar la frecuencia de sobrepeso y obesidad con el género, se encontró que del género femenino, un $37,7 \%$ tenían sobrepeso u obesidad, en cuanto al género masculino, un 34,3\% tenían sobrepeso u obesidad.

En cuanto a la prevalencia de sobrepeso y obesidad y el tipo de escuela (pública o privada), se encontró que los niños de escuelas privadas presentaron mayor frecuencia de sobrepeso y obesidad (46,3\%) en relación a las escuelas públicas (33,2\%). Se encontró asociación entre el tipo de escuela y el sobrepeso y obesidad (análisis simple en tabla de $2 \times 2 ; p<0,05)$ (Tabla 2). Con respecto a los alimentos que los escolares consumen en la tabla 3 se representan los alimentos que consumen con mayor frecuencia durante las meriendas.

\section{TABLA 1}

Características Sociodemográficas de los Escolares

\begin{tabular}{|ccc|}
\hline & fr & \% \\
\hline Género & & \\
Femenino & 191 & 53,5 \\
Masculino & 166 & 46,5 \\
Edad en años & & \\
6 & 50 & 14,0 \\
7 & 66 & 18,5 \\
8 & 69 & 19,3 \\
9 & 62 & 17,4 \\
10 & 47 & 13,1 \\
11 & 63 & 17,7 \\
Tipo de escuela & & \\
Pública & 277 & 77,6 \\
Privada & 80 & 22,4 \\
Grado escolar & & \\
Primero & 39 & 10,9 \\
Segundo & 94 & 26,3 \\
Tercero & 71 & 19,8 \\
Cuarto & 58 & 16,3 \\
Quinto & 60 & 16,9 \\
Sexto & 35 & 9,8 \\
\hline TOTAL & $\mathbf{3 5 7}$ & $\mathbf{1 0 0 , 0}$ \\
\hline
\end{tabular}

En lo referente a los principales alimentos que los escolares consumieron en las 24 horas previas al estudio, los más frecuentes fueron los siguientes: huevos $78,7 \%$; leche $72,0 \%$; frijoles $70,6 \%$; arroz $66,4 \%$; refrescos o gaseosas $65,3 \%$; mantequilla $63,3 \%$; carne de pollo $62,2 \%$; pan blanco $61,9 \%$; aceite $60,8 \%$; churros (golosinas) $58,8 \%$; jugos envasados $58,3 \%$; plátano $56,0 \%$; tomate $55,7 \%$; pan dulce $55,2 \%$; queso $52,9 \%$.

Se compararon los hábitos y prácticas alimentarias de los escolares entre los centros educativos públicos y privados, en cuanto a la merienda se encontró que 53,4\% de los estudiantes de escuelas públicas y $36,3 \%$ de las escuelas privadas consumen merienda elaborada en casa y además, reciben dinero para comprar alimentos en la escuela. Al preguntar sobre la cantidad de dinero gastado en la merienda, la media que recibieron en las escuelas públicas fue \$ 0,5062 (12 lempiras) y \$ 0,8016 (19 lempiras) para las privadas. Con respecto al número de comidas que consumen al día, la media en ambos grupos fue de 4 cuatro comidas diarias, en las tablas 4 y 5 se describen otros hábitos y practicas estudiadas.

En relación a las actividades de descanso que realizan, se encontró que en ambos grupos, un $72 \%$ de los escolares duermen durante la noche de 8 a 12 horas al día, un 24,9\% duerme menos de 8 horas y un 3,1\% más de 12 horas. En cuanto a las horas que descansan durante el día $85,4 \%$ descansa menos de 8 horas durante el día, un 7,3\% de 8 -12 horas y un 7,3\% más de 12 horas. En lo que respecta al tiempo en que permanecen sentados, un $97,2 \%$ permanece menos de 6 horas sentadas y $2,8 \%$ de 6 a 10 horas. En cuanto al tiempo que permanecen viendo televisión, la computadora o jugando frente a pantallas de video se encontró que $88,5 \%$ permanecen menos de 6 horas realizando estas actividades, un 9,8\% entre $6-10$ horas y un 1,7\% permanecen más de 10 horas sentados frente a una pantalla. En lo referente a caminar ambos grupos de escolares caminan menos de 5 cuadras al día 59,7\%, de 5-15 cuadras $26,6 \%$ y más de 15 cuadras $13,7 \%$. En cuanto a la actividad física y ejercicios o deportes programados se encontró diferencia de frecuencia entre los estudiantes de ambos tipos de escuelas (Tabla 6).

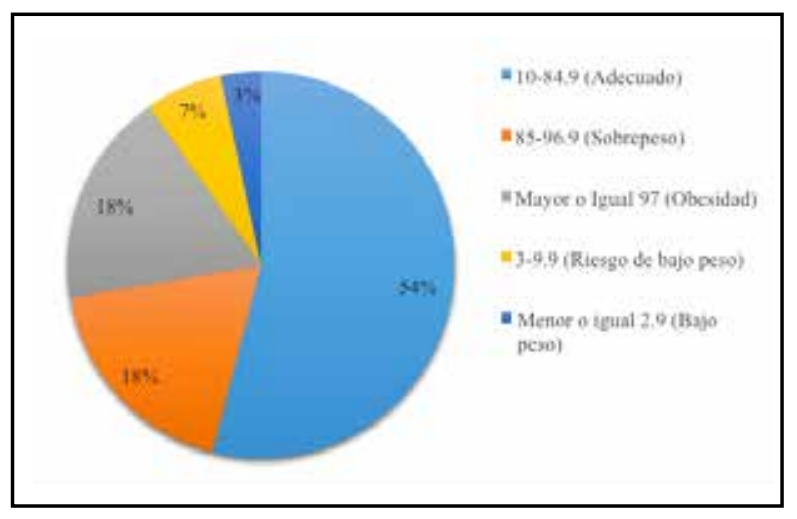

Gráfico 1. Diagnóstico nutricional según percentil para IMC (OMS) 
TABLA 2

Prevalencia de sobrepeso y obesidad de acuerdo al tipo de establecimiento educacional al que asiste.

\begin{tabular}{|c|c|c|c|}
\hline \multicolumn{4}{|c|}{ Sobrepeso u obesidad } \\
\hline Tipo de escuela & $\mathrm{Si}$ & No & Total \\
\hline Privada & 37 & 43 & 80 \\
\hline & $46,3 \%$ & $53,8 \%$ & $100 \%$ \\
\hline Pública & 92 & 185 & 277 \\
\hline & $33,2 \%$ & $66,8 \%$ & $100 \%$ \\
\hline Total & 129 & 228 & 357 \\
\hline Tabla $2 \times 2 ; p<0,05^{*}$ & & & \\
\hline
\end{tabular}

TABLA 3

Alimentos consumidos por los escolares en el tiempo de merienda.

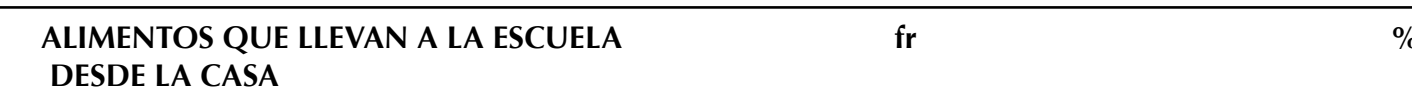

Bebidas o jugos azucarados

Agua

Aperitivos salados (snacks), papas fritas, etc.

Frutas

Dulces, galletas, chocolates, etc.

Donas, pastel, pan dulce, etc.

No aplica, no traigo merienda de la casa

Verduras

Bebidas o jugos sin azúcar (jugos naturales)

Leche o yogurt

Emparedados o similares

Baleadas o similares

Otras

\begin{tabular}{rr}
146 & 40,9 \\
138 & 38,7 \\
114 & 31,9 \\
109 & 30,5 \\
103 & 28,9 \\
61 & 17,1 \\
49 & 13,7 \\
43 & 12,0 \\
38 & 10,6 \\
25 & 7,0 \\
13 & 3,6 \\
11 & 3,1 \\
6 & 1,7 \\
\hline
\end{tabular}

$\%$

$\%$

Bebidas o jugos azucarados

162

154

130

79

74

33

32

27

9

7

4

4

3
45,4

43,1

36,4

22,1

20,7

9,2

9,0

7,6

2,5

2,0

1,1

1,1

0,8
Emparedados o similares

*Los participantes podían contestar más de una opción 


\section{TABLA 4}

Hábitos y prácticas alimentarias de los escolares de establecimientos públicos y privados.

Hábito o práctica

Vasos de agua diarios

4 o menos vasos

5 o más vasos

Platos de verdura diaria

1 plato

2 platos

3 o más platos

No consumo

Frutas diarias

1 fruta

2 frutas

3 frutas

4 frutas

5 o más frutas

No consumo

Porción de lácteos al día

1 porción

2 porciones

3 porciones

4 porciones

5 o más porciones

No consumo

Pan al día

1 pan

2 panes

3 o más panes

No consumo

\section{Comida rápida por semana}

1 día a la semana

2 días a la semana

3 o más días a la semana

No consumo

Pescado por semana

1 día a la semana

2 días a la semana

3 o más día a la semana

No consumo
Escuelas Públicas

fr $\%$

73,3

26,7

61

19

76,3

74

177

44

30

26

63,9

15,9

10,8

9,4

39

26

5

10

32,5

6,3

12,5

33,2

28,8

30,3

23

36,3

15,9

7,2

5,4

7,9

29

18,8

6,3

3,8

6,3

32,1

46,2

13,4

3,6

2,2

2,5

40,8

26,0

14,4

18,8

26

37

32,5

46,3

7,5

7,5

2,5

3,8

3

47,5

26,3

16,3

10,0 


\section{Refresco o gaseosa al día}

$\begin{array}{lrr}1 \text { vaso } & 138 & 49,8 \\ 2 \text { vasos } & 65 & 23,5 \\ 3 \text { o más vasos } & 62 & 22,4 \\ \text { No consumo } & 12 & 4,3\end{array}$

49,8

23,5

22,4

4,3

25,3

15,9

21,3

32,9

4,7
27

31

16

6

33,8

38,8

20,0

7,5

Helados y golosinas dulces por semana

1 día de la semana

2 días de la semana

3 días de la semana

4 o más días de la semana

No consumo

\section{Golosinas saladas por semana}

1 día a la semana

2 días a la semana

3 días a la semana

4 o más días a la semana

No consumo

$\begin{array}{rr}70 & 25,3 \\ 44 & 15,9 \\ 59 & 21,3 \\ 91 & 32,9 \\ 13 & 4,7\end{array}$

\section{0}

40

48

123

16
18,1

14,4

17,3

44,4

5,8
20

20

4

29

7

25

10

10

27

8

25,0

25,0

5,0

36,3

8,8

31,3

12,5

12,5

33,8
10,0

\section{Consume fruta durante merienda}

\section{A veces}

Nunca

Siempre

TOTAL

277

53,8

40,1

6,1

\section{8}

15

7

100

80

72,5

18,8

8,8

\section{TABLA 5}

Prácticas en la elaboración de los alimentos de los escolares de establecimientos públicos y privados

Práctica

Elaboración de merienda

Elaborada en casa y no recibe dinero

No come merienda

Recibe dinero para comprarla

Elaborada en casa y recibe dinero

Lugar donde desayuna

En la casa y la escuela

En la escuela

En su casa

No desayuna

Lugar donde Almuerza

En la casa y la escuela

En la escuela

En otro lugar

Solo en casa

No almuerza

\section{Escuelas Públicas}

fr

$\begin{array}{rr}56 & 20,2 \\ 6 & 2,2 \\ 67 & 24,2 \\ 148 & 53,4\end{array}$

$10 \quad 3,6$

$17 \quad 6,1$

$242 \quad 87,4$

$8 \quad 2,9$

$22 \quad 7,9$

$35 \quad 12,6$

$1 \quad 0,4$

$214 \quad 77,3$

5
Escuelas privadas

fr

$\%$

TOTAL

277

100,0

33

3

15

29

6

25

46

3

18

25

1

36

0
41,3

3,8

18,8

36,3

7,5

31,3

57,5

3

22,5

31,3

1,3

45,0

0,0 


\section{TABLA 6}

Antecedentes de actividad física de los escolares de establecimientos públicos y privados.

\begin{tabular}{|ccccc|}
\hline Actividad física & \multicolumn{2}{c|}{ Escuelas Públicas } & \multicolumn{2}{c|}{$\begin{array}{c}\text { Escuelas privadas } \\
\mathbf{f r}\end{array}$} \\
& $\mathbf{f r}$ & & & \\
\hline Ejercicio o deporte programado & & & 44 & 55,0 \\
Menos de 2 horas por semana & 170 & 61,4 & 28 & 35,0 \\
De 2-5 horas por semana & 84 & 30,3 & 8 & 10,0 \\
Más de 5 horas por semana & 23 & 8,3 & & 31,3 \\
& & & 25 & 40,0 \\
Actividades o juegos al aire libre & 115 & 41,5 & 23 & 28,7 \\
Menos de 30 minutos al día & 86 & 31,1 & & $\mathbf{1 0 0 , 0}$ \\
30-60 minutos diarios & 76 & 27,4 & $\mathbf{8 0}$ & \\
\hline Más de 60 minutos al día & $\mathbf{2 7 7}$ & $\mathbf{1 0 0 , 0}$ & & \\
\hline
\end{tabular}

\section{DISCUSIÓN}

Entre los factores asociados a el sobre peso y obesidad se encuentra el consumo de alimentos con alto contenido calórico y azucares refinadas, en la Región ha aumentado la publicidad de bebidas azucaradas y productos de alto contenido calórico y de bajo valor nutricional dirigida a la niñez, esto influye en sus preferencias alimentarias, lo que piden comprar y en sus hábitos alimentarios, provocando una preferencia por comida de poco valor nutritivo en lugar de comida saludables ${ }^{19}$. Aunque no se estudió el fenómeno de la publicidad, la influencia de esta en los hábitos alimentarios puede estar generando un impacto en nuestro medio, debido a que se observó que los alimentos más frecuentes consumidos que llevaban de su casa los escolares de instituciones tanto públicas como privadas durante la merienda fueron: bebidas o jugos azucarados con 40,9\%; el segundo lugar lo ocupó el agua con 38,7\%, seguido de golosinas tipos "snacks" y salados con 31,9\% y papas fritas y churros con $30,5 \%$.

Esto tiene una relación con el nivel socioeconómico, en este estudio se encontró que los niños de escuelas privadas de la comunidad estudiada tienen mayor prevalencia de sobrepeso y obesidad $37(46,3 \%)$ que los de las escuelas públicas $129(33,2 \%)$ y se encontró asociación entre el tipo de escuela y el sobrepeso y obesidad con significancia estadística $(p=0,04)$. Esto puede relacionarse con la mayor posibilidad de obtener alimentos ricos en carbohidratos y lípidos, también se observó que entre los niños que llevaban dinero para comprar merienda la media de dinero en las escuelas privadas fue de 19 Lempiras mayor que en las escuelas públicas de 12 Lempiras.
El contraste del nivel socioeconómico con la obesidad infantil, también se ha evaluado estudios previos en Honduras como el realizado en el IHSS en el año $2002^{20}$, en donde se encontró que el $27,7 \%$ de los niños con nivel socioeconómico alto, $58,4 \%$ estrato medio y $13,9 \%$ estrato bajo con sobrepeso y obesidad. Además, se ha descrito que el nivel socioeconómico tiene una fuerte influencia con el consumo de alimentos fuera del hogar como: golosinas, frutas, verduras, jugos y bebidas azucaradas y el desarrollo de sobrepeso ${ }^{13}$. En relación a los alimentos que compran en la escuela, se observó, que consumen alimentos similares a los que traen de su casa: el $45,4 \%$ bebidas o jugos azucarados, 43,1\% churros, papas fritas, "snack", dulces, galletas y chocolates.

Lo anteriormente mencionado actúa como un ambiente obesogénico y es uno de los principales factores que contribuyen al aumento del sobrepeso y la obesidad, es decir, la suma de factores genéticos más las influencias del entorno ${ }^{21}$, o las condiciones de vida que contribuyen a la obesidad en individuos o comunidades y que simultáneamente actúan como barreras para mantener un peso saludable. Otro factor importante es la actividad física, aunque se trata de un fenómeno complejo y multicausal, el tiempo dedicado a ver televisión se ha asociado de manera consistente con la obesidad infantil en estudios transversales, longitudinales e intervenciones preventivas ${ }^{10,22,23}$.

Los hábitos alimentarios, sedentarios y la actividad física son los factores culturales y ambientales que tienen mayor relevancia en la contribución con el riesgo de sobrepeso y obesidad ${ }^{24}$. En este estudio se observó que el $11,5 \%$ de los escolares pasan 6 o más horas diarias frente 
al televisor o computador y $88,5 \%$ menos de 6 horas, también se encontró que el 97,2\% de los escolares están sentados realizando sus tareas, leyendo dibujando menos de 6 horas diarias. Los niños menores de 11 años de edad se recomienda que duerman 10 horas $^{19,25,26}$. Este estudio reveló que la mayoría ( $72 \%$ ) de los niños de ambos grupos de escuelas, públicas y privadas, duermen durante la noche de 8 a 12 horas al día. Por lo que estos escolares cumplen con esta recomendación para un correcto crecimiento y desarrollo. En una revisión bibliográfica realizada en el 2011 se encontró que los niños dedican un promedio de 135 minutos sin actividad o en estado sedentario, siendo discretamente mayor en los varones ${ }^{9}$.

La actividad física desempeña un papel importante en la prevención de la obesidad ${ }^{1}$ y cuando se combinan actividad física reducida y un estilo de vida sedentario constituyen factores de riesgo de obesidad, insulino-resistencia y cardiovascular $^{18}$. El 59,9\% de los escolares que participaron en este estudio hacen ejercicios o deportes programados menos de 2 horas al día, el 59,7\% de los escolares caminan menos de 5 cuadras para llegar a sus escuelas. En las escuelas públicas el $41,5 \%$ de escolares realizan juegos al aire libre menos de 30 minutos al día, mientras que el $40 \%$ de los niños de escuelas privadas realizan entre 30 y 60 minutos de juegas al aire libre. Dedicar pocas horas de actividad física al día es un factor asociado para que los niños aumenten de peso con el riesgo de llegar a sobrepeso u obesidad.

Está establecido que si ambos progenitores son obesos el riesgo para la descendencia es del $80 \%$, cuando sólo uno de los progenitores lo es, el riesgo desciende al $40 \%$, y si ninguno de los progenitores es obeso, el riesgo en sus hijos queda en el $3-7 \%{ }^{27}$. En este estudio no se interrogo sobre el antecedente de sobrepeso y obesidad en los familiares de los niños que presentaron estas condiciones, por lo tanto, se sugiere realizar otro estudio con esta orientación.

El sobrepeso y la obesidad infantil tienen consecuencias psicosociales en los escolares obesos, en donde son objeto temprano y sistemático de discriminación por sus pares, a esto se suma la poca habilidad para desarrollar actividades deportivas o juegos, lo cual aumenta esta discriminación y rechazo ${ }^{25}$. En vista de lo anterior, para combatir la obesidad infantil, la OMS aboga por una alimentación saludable que debe basarse en el consumo diario de frutas y verduras, cereales integrales, legumbres, leche, pescado y aceite vegetal, con poco consumo de carnes rojas y procesadas. Los alimentos naturales e integrales son la piedra angular de la cocina tradicional en la Región de las Américas ${ }^{19,26}$, sin embargo, a pesar de la facilidad de adquisición de alimentos naturales para los escolares se observó que solo el $6,1 \%$ de los estudiantes de escuelas privadas y $8,8 \%$ de escuelas públicas consume frutas durante la merienda escolar diariamente. El 9,4\% de escolares de centros públicos y $12,5 \%$ de escuelas privadas afirmaron no consumir verduras regularmente, la mayoría lo realizaba solamente 1 vez al día (63,9\% niños de escuelas públicas y el $48,8 \%$ de escuelas privadas). Resultados similares también fueron observados en otros estudios como el realizado en Chile donde los niños presentaban un bajo consumo de verduras y frutas $^{26}$ y el estudio realizado en Venezuela donde encontraron una baja frecuencia de consumo de frutas y vegetales ${ }^{17}$. Por lo que se puede establecer que dicha recomendación de una alimentación saludable de la OMS necesita ser implementada con más diligencia ya que es un fenómeno que se ha reportado en estudios en diferentes ciudades de la región.

La relación entre la malnutrición, la infección, enfermedad y la pobreza, y la relación entre la obesidad, las enfermedades crónicas y el bienestar económico, ya no son aplicables en los países desarrollados y se están reduciendo todos los días. En las zonas periurbanas es normal encontrar una familia en la que el padre tenga hipertensión arterial, puede tener sobrepeso o no, es estatura baja, y Tiene una probable historia de desnutrición; La madre es anémica, probablemente es obesa y corta; Y los niños sufren infecciones frecuentes $y$ Muestran retraso del crecimiento ${ }^{28}$. En Honduras un país de bajo nivel socioeconómico, el sobrepeso y la obesidad se da más en los niveles medio- alto, desde el punto de vista socioeconómico y de ingreso.

\section{CONCLUSIONES}

El sobrepeso y la obesidad infantil son un problema de han presentado aumento de la prevalencia nuestro medio. Los estudiantes de escuelas privadas de la comunidad estudiada presentaron mayor prevalencia de sobrepeso y obesidad que los de centros educativos públicos. Así mismo reportaron mayor dinero para la compra de alimentos en la merienda escolar que los de las escuelas públicas. Los escolares de la comunidad estudiada están consumiendo entre los principales alimentos productos con alto contenido calórico y azucares refinadas, especialmente en las meriendas escolares. La actividad física de los escolares estudiados fue adecuada en la mayoría de los casos. No existe diferencia de género entre los niños que presentaron sobrepeso u obesidad.

Conflictos de interés: los autores declaran que no existe conflicto de interés

\section{Financiamiento: Autofinanciado}

\section{RESUMEN}

Objetivo: Comparar los factores asociados de sobrepeso y obesidad en los niños de escuelas públicas y privadas de la colonia Kennedy, Tegucigalpa, Honduras. Materiales y Métodos. Se estudiaron 357 escolares entre 6 y 11 años. Se realizó evaluación nutricional, frecuencia de consumo, hábitos y prácticas alimentarias y actividad física mediante encuesta. Se excluyeron escolares con sobrepeso y obesidad secundaria a una patología de base. La información fue analizada con el programa Epi-info, seguido de análisis variado y multivariado. Resultados: Se encontró un 18\% de escolares con obesidad y $18 \%$ con sobrepeso. Los niños 
de escuelas privadas presentaron mayor frecuencia de sobrepeso y obesidad (46,3\%) que los de escuelas públicas $(33,2 \%)$. Más de la mitad (53,4\%) de los estudiantes de escuelas públicas y $36,3 \%$ de las escuelas privadas consumieron consumen merienda elaborada en casa. Un 58,5\% de estudiantes de escuelas públicas y un 68,7\% de centros privados, realizaron algún tipo de actividad física al aire libre. Conclusión: El sobrepeso y la obesidad infantil son un problema de salud prevalente. En nuestra muestra, los estudiantes de escuelas privadas tenían mayor sobrepeso y obesidad que los de escuelas públicas. La actividad física realizada por los escolares de escuelas públicas y privadas fue adecuada en la mayoría de los casos.

Palabra Clave: Nutrición Infantil; Alimentación; Dieta; Obesidad Infantil; Sobrepeso.

\section{BIBLIOGRAFÍA}

1. World Health Organization. World strategy on food regime, physical activity and health. Geneva: WHO; 2004. http:// www.who.int/dietphysical activitchildhood_what/es/index. html

2. Pan American Health Organization. Countries of the Americas commit to adopt measures to reduce childhood obesity. Washington, D.C. OPS. http://www.paho.org/hq/index. php?option $=$ com_content $\&$ view $=$ article $\& i d=10057 \% 3$ A2014countries-pledge-action-to-reduce-child-obesity-in-the-americas\& Itemid $=1926 \&$ lang $=\mathrm{es}$

3. Arias-Segura JO. Childhood Obesity. Luxmédica. 2012; 7(21): 27-46. http://www.uaa.mx/direcciones/dgdv/editorial/docs/ luxmedica21.pdf

4. Pastrana Franco I. Obesity in school children: family prevalence and its influences [thesis]. Valladolid: University of Valladolid. Valladolid Nursing Faculty; 2014. Available in: http://uvadoc. uva.es/handle/10324/5227

5. Lizardo A. E, Díaz A. Overweight and Childhood Obesity. Rev. Med Hondur 2011; 79(4): 208-213.

6. Schmidt-RioValle J, González-Jiménez E, García Villamil SS, Correa Bautista JE, Mancipe Navarrete JA, Meneses-Echávez $J F$. Effectiveness of educational interventions performed in Latin America for the prevention of overweight and childhood obesity in school children from 6 to 17 years old: a systematic review. Nutr Hosp. 2015; 31(1): 102-114.

7. Lobos Fernández LL, Leyton Dinamarca B, Kain Bercovich J, Vio del Río F. Evaluation of an educational intervention for the prevention of childhood obesity in basic schools of Chile. Nutr. Hosp 201328(4)1156-1164. http://scielo.isciii.es/pdf/nh/ v28n4/26original17.pdf

8. Álvarez-Dongo D, Sánchez-Abanto J, Gómez-Guizado G, Tarqui-Mamani C. Overweight and obesity: prevalence and social determinants of overweight in Peruvian population (2009-2010). Rev Perú Med Exp Salud Pública 2012; 29(3): 303-313.

9. Villagrán Pérez S, Rodríguez-Martín A, Novalbos Ruiz JP, Martínez Nieto JM, Lechuga Campoy LL. Habits and modifiable lifestyles in overweight and obese children. Nutr Hosp. 2010; 25(5): 823-831.

10. Félix-Rivera M. Obesity in poverty conditions. Epidemiological study in public school children of Tegucigalpa, Honduras, 2000. Rev. Med Hondur. 2000; 73: 10-14.

11. Rodríguez Melián A, Álvarez González LM, García Melián CM, Mariné Alonso M de A. Evaluation of the nutritional condition in children of the community "Los Naranjos", Carabobo, Venezuela. Rev Cubana Hig Epidemiol. 2012; 50(3): 268-277.

12. Puente Perpiñán M, Ricardo Falcón TR, Fernández Díaz RR. Risk factors related to obesity in girls and boys under 5 years old. MEDISAN 2013; 17(7): 1065-1071.

13. Shamah-Levy T, Cuevas-Nasu L, Méndez-Gómez-Humarán I, Jimenez-Aguilar A, Mendoza-Ramírez AJ, Villalpando S. Obesity in Mexican children at school ages is associated with consumption of food outside the home: during the journey from home to school. Arch Latinoam Nutr. 2011; 61(3): 288295.

14. Restrepo SL. The family and its role in the formation of eating habits in school children. An approach to everyday life. Bol Antropol. 2005;19(36): 127-148.

15. Sampieri R, Fernández C, Baptista P. Metodología de la investigación. 5ta. ed. D.F., México: McGraw Hill;2010.

16. Lera L, Fretes G, González CG, Salinas J. Validity of an instrument for assessing food consumption, food habits and cooking skills in 8-11years old students. Nutr Hosp. 2015;31(5):1977-1988.

17. Coromoto-Nava BM, Pérez-G A, Herrera HA, Hernández-H RA. Anthropometric-nutritional assessment, dietary habits and physical activity in preschool children. Rev Chil Nutr. 2011; 38(3): 301-304.

18. Godard C, Rodríguez M del P. Díaz N, Lera L, Salazar G, Burrows R. Value of a clinical test to assess physical activity in children. Rev Méd Chile. 2008; 136: 1155-1162.

19. Pan American Health Organization. Action plan for the prevention of obesity in childhood and adolescence. Washington D.C. OMS; 2014 http://www.paho.org/hq/index. php?option=com_docman\&task=doc_view\&ltemid=270\&gid $=28899 \&$ lang=es

20. Girón K, Espinoza L. Obesity Epidemiology in the Out Patients of the Endocrinology Pediatrics Department. Specialty Hospital of the Honduran Social Security Institute (IHSS). Period 20022004. Rev. Med. de los Post Grados de Medicina UNAH. 2006; 9(2): 243-248.

21. Saavedra IM, Dattilo AM. Food and dietary factors associated with childhood obesity: recommendations for prevention before two years of life. Rev. Perú Med Exp Salud Pública. 2012; 29(3): 379-85.

22. Ocampo T. Prada G. Herrán F. Food consumption patterns and overweight excess in children; survey of the nutritional situation in Colombia, 2010. Rev Chil Nutr. 2014; 41(4): 351359.

23. Loaiza M, Atalah SE. Risk Factors for obesity in school children of first basic year of Punta Arenas. Rev. Chil. pediatr. 2006; 77(1): 20-26.

24. Villagran Pérez S, Novalbos-Ruiz JP, Rodriguez-Martín A, Martínez-Nieto JM, Lechuga-Sancho AM. Implications of family socioeconomic level on risk behaviors in child-youth obesity. Nutr Hosp. 2013; 28(6): 1951-1960.

25. Sánchez Echenique M. Epidemiological aspects of Childhood Obesity. Rev Pediatr Aten Primaria Supl. 2012 ;14(21): 9-14

26. Manuel Hernández, M. Triana, Ruiz Álvarez, V. Obesity, a worldwide epidemic. Implications of genetics. Rev. Cubana Invest Bioméd. 2007;26(2):1-10Guerra Cabrera C, Vila Díaz J, Apolinaire J, Cabrera Romero A, Santana Carbolla I, Almaguer Sabina P. Risk Factors associate with overweight and obesity in adolescents. Medisur. 2009; 7(2). http://scielo.sld.cu/scielo. php?script=sci_arttext\&pid=S1727-897X2009000200004

27. Obesity and Poverty. A new Public Health Challenge. Ed. Manuel Peña y Jorge Bacallao (Eds.). Washington PAHO. Publicación Científica No 576. 2000. 